Revue internationale P.M.E.

Économie et gestion de la petite et moyenne entreprise

\title{
Stratégies de croissance externe des moyennes entreprises patrimoniales sous-traitantes
}

\section{Natacha Tréhan}

Volume 17, numéro 1, 2004

URI : https://id.erudit.org/iderudit/1008450ar

DOI : https://doi.org/10.7202/1008450ar

Aller au sommaire du numéro

Éditeur(s)

Presses de l’Université du Québec

ISSN

0776-5436 (imprimé)

1918-9699 (numérique)

Découvrir la revue

Citer cet article

Tréhan, N. (2004). Stratégies de croissance externe des moyennes entreprises patrimoniales sous-traitantes. Revue internationale P.M.E., 17(1), 9-35.

https://doi.org/10.7202/1008450ar
Résumé de l'article

De très nombreuses études ont traité des stratégies de croissance externe des grandes entreprises cotées, alors que les firmes de moyenne dimension ont retenu bien peu d'attention. L'objectif de cet article est de relever, de hiérarchiser puis d'analyser les facteurs qui vont inciter ou dissuader une moyenne entreprise patrimoniale sous-traitante d'entreprendre une démarche de croissance externe.

Les trois principaux paramètres d'une décision stratégique sont considérés dans cette analyse : les aspirations du dirigeant-propriétaire, le potentiel de l'entreprise et les exigences de son environnement.

L'étude, réalisée à partir d'un questionnaire publiposté, porte sur 147 entreprises françaises non cotées. Les méthodes d'analyses de données sont explicatives : il s'agit essentiellement de régressions logistiques.

Les résultats obtenus amènent à s'interroger sur la dimension hétéronome de l'action stratégique des moyennes entreprises sous-traitantes au vu des nouvelles exigences de leurs donneurs d'ordres en matière d'achats et d'approvisionnement. 


\title{
Stratégies de croissance externe des moyennes entreprises patrimoniales sous-traitantes
}

\author{
Natacha TRÉHAN \\ École supérieure des affaires - ESA \\ Université Pierre-Mendès-France, Grenoble II
}

MOTS CLÉS

Relations donneur d'ordres et sous-traitants
Entreprises patrimoniales - Aspirations du dirigeant-propriétaire
Croissance externe - Entreprises sous-traitantes
Politiques achats-approvisionnement

\section{RÉSUMÉ}

De très nombreuses études ont traité des stratégies de croissance externe des grandes entreprises cotées, alors que les firmes de moyenne dimension ont retenu bien peu d'attention. L'objectif de cet article est de relever, de hiérarchiser puis d'analyser les facteurs qui vont inciter ou dissuader une moyenne entreprise patrimoniale sous-traitante d'entreprendre une démarche de croissance externe.

\section{L'AUTEURE}

NATACHA TRÉHAN est maître de conférences à l'École supérieure des affaires, Université PierreMendès-France, Grenoble II, où elle est aussi responsable du DESS de management des achats. Elle enseigne le management stratégique et les stratégies achats. Elle est également chercheure au CERAG, Centre d'études et de recherches appliquées à la gestion, UMR CNRS 5820. Ses axes de recherche sont les entreprises patrimoniales, les relations entre donneurs d'ordres et sous-traitants, le management stratégique des moyennes entreprises patrimoniales et les stratégies de croissance externe. Adresse: École supérieure des affaires, ESA, Université Pierre-Mendès-France, Grenoble II, B.P. 47, 38040 Grenoble, Cédex 9, France. Téléphone : 334768278 55. Courriel : <natacha.trehan@esa.upmf-grenoble.fr>. 
Les trois principaux paramètres d'une décision stratégique sont considérés dans cette analyse: les aspirations du dirigeant-propriétaire, le potentiel de l'entreprise et les exigences de son environnement.

L'étude, réalisée à partir d'un questionnaire publiposté, porte sur 147 entreprises françaises non cotées. Les méthodes d'analyses de données sont explicatives: il s'agit essentiellement de régressions logistiques.

Les résultats obtenus amènent à s'interroger sur la dimension hétéronome de l'action stratégique des moyennes entreprises sous-traitantes au vu des nouvelles exigences de leurs donneurs d'ordres en matière d'achats et d'approvisionnement.

\section{ABSTRACT}

Many works have been produced in the field of external growth strategy concerning large quoted companies. Medium-sized firms have focused few attention. This work aims to identify, to organize into a hierarchy, then to analyze factors which are going to incite or, on the contrary, to dissuade a patrimonial medium-sized subcontractor to undertake an external growth strategy.

The three essential parameters of a strategic decision are considered in this analysis : aspiration of the leader-owner, potential of the company and requirements of its environment.

The empirical study, based on the mailshot of a questionnaire, concerns 147 non-quoted French companies. Analytical methods of data are explanatory: it is essentially about logistic regressions.

Obtained results lead to question about the constrainted dimension of the strategic action of the medium-sized subcontractors in view of the new purchasing and supply requirements of their principals.

\section{RESUMEN}

Numerosas aportaciones han sido realizadas sobre las estrategias de crecimiento externo de las grandes empresas que cotizan en Bolsa. Sin embargo, las medianas empresas han sido objeto de poca atención. El objeto de este artículo es identificar, jerarquizar y analizar los factores que van a empujar o, por el contrario, disuadir a una mediana empresa subsidiaria a emprender una estrategia de credimiento interno.

Los tres parámetros esenciales de una decisión estratégica están considerados en este análisis : las aspiraciones del dirigente-propietario, el potencial de la empresa, las exigencias de su entorno.

El estudio, realizado a partir de un cuestionario enviado por correo, ha sido llevado a cabo sobre 147 empresas francesas que no cotizan en el mercado de valores. Los métodos de análisis de datos son explicativos, se trata esencialmente de regresiones logísticas.

Los resultados obtenidos llevan a preguntarse sobre la dimensión heteronómica de la acción estratégica de las medianas empresas subsidiarias a la vista de las nuevas exigencias de sus responsables en materia de compras y aprovisionamientos. 


\section{ZUSAMMENFASSUNG}

Zahlreiche Beiträge sind über externe Wachstumsstrategien der großen kotierten Unternehmen verwirklicht worden. Die Unternehmen mittlerer Grösse wurden bislang kaum beachtet. Dieser Artikel bezweckt, die Faktoren zu identifizieren, zu analysieren und zu ordnen, welche ein mittleres Unternehmen anspornen eine externe Wachstumsstrategie als Zulieferer auszuführen.

Drei wesentlichen Parameter für eine strategische Entscheidung wurden in dieser Analyse betrachtet: die Aspirationen des Manager-Eigentümers; das Potential der Unternehmung; die Anforderungen seiner Umwelt.

Die Studie wurde realisiert mittels einem Fragebogen bei 147 nicht kotierten französischen Unternehmen. Die Analysemethoden sind erklärend, wobei es sich hauptsächlich um logistische Regressionen handelt. Die erzielten Ergebnisse beziehen sich auf Befragungen der strategischen Aktion der mittleren Unternehmen, die angesichts der neuen Anforderungen seitens der Auftraggeber, als Zulieferer den Materieleinkauf und die Versorgung ausführen.

\section{Introduction}

Les recherches ayant trait aux stratégies de croissance externe des grandes entreprises sont très nombreuses. S'agissant des firmes de moyenne dimension, elles sont le plus souvent appréhendées comme des cibles potentielles pour ces grandes sociétés (Schwartz, 1982) et non comme des acteurs d'opérations de fusions, d'acquisitions, etc.

Contrairement à ce que l'on relève pour les grandes entreprises cotées, les conditions et modalités de la croissance externe qui ont cours dans les entreprises patrimoniales de moyenne dimension, non cotées, ont fait l'objet de très peu de travaux récents et demeurent mal connues. Pourtant, le sujet mérite attention: à partir d'un dépouillement quasi systématique de la presse économique française, entre 1992 et 1999, nous avons relevé près d'un millier d'entreprises patrimoniales françaises, de moyenne dimension, ayant réalisé au moins une opération durant cette période.

La présente étude est née d'un double constat:

- d'une part, lors de la constitution de notre base de données, il est apparu que la moitié de ces entreprises étaient sous-traitantes (49\% des 147 entreprises de notre échantillon);

- d'autre part, lors d'entretiens exploratoires réalisés avec des dirigeantspropriétaires, il nous a semblé que les nouvelles exigences des donneurs d'ordres constituaient un facteur incitatif clé de leurs stratégies de croissance externe, à tel point que nous pouvions nous demander si la croissance externe des entreprises sous-traitantes est véritablement vécue comme 
une stratégie «délibérée », au sens littéral du terme, ou plutôt comme une stratégie «contrainte» par la menace implicite du déréférencement ou du non-référencement.

La stratégie de toute entreprise s'alimente et se construit de ses interactions avec l'environnement. Si cet environnement est contraignant et que la stratégie est commandée de l'extérieur, on parlera de stratégie hétéronome (Koenig, 1996) ou stratégie contrainte. La problématique de cette recherche est donc la suivante : les stratégies de croissance externe des entreprises patrimoniales sous-traitantes, de moyenne dimension, relèvent-elles de stratégies hétéronomes ou contraintes?

Pour tenter de répondre à cette interrogation, nous nous proposons de relever et de hiérarchiser les facteurs qui vont inciter ou, au contraire, dissuader une moyenne entreprise patrimoniale sous-traitante d'entreprendre une stratégie de croissance externe. Comme une décision de croissance externe relève typiquement d'un problème de management stratégique, dans la lignée des travaux d'Ansoff, nous nous intéressons aux trois paramètres essentiels d'une décision stratégique :

- les aspirations de la coalition au pouvoir, leur projet politique, en l'occurrence ceux du dirigeant-propriétaire dans une moyenne entreprise patrimoniale ;

- le potentiel de l'entreprise (ses compétences et ses ressources);

- les exigences de l'environnement, en particulier celles des donneurs d'ordres.

Il convient, au préalable, de préciser certains termes utilisés. La moyenne entreprise patrimoniale (MEP) se caractérise par deux principaux types d'actionnaires, personnes physiques, contrôlant majoritairement le capital et participant activement à la direction et à la gestion de l'entreprise. Soit «l'entrepreneur » est le propriétaire quasi unique : son entreprise est alors de nature «personnelle pure» (l'entreprise personnelle peut aussi être caractérisée par un entrepreneur dirigeantpropriétaire majoritaire, plus un ou plusieurs associés, personnes physiques, minoritaires), soit ce sont les membres d'une même famille qui contrôlent majoritairement le capital et participent activement à la direction. Nous considérons avec Déry et al. (1993) que les liens de parenté peuvent être du second degré et plus. La MEP se définit donc par une structure de propriété patrimoniale et par une interdépendance famille-entreprise. Elle est juridiquement indépendante (il ne s'agit ni d'une filiale d'un grand groupe ni d'un établissement); sa gestion est autonome. La transférabilité de ses droits de propriété est faible dans la mesure où nous excluons les entreprises cotées. Enfin, ne sont retenues que les entreprises dont l'effectif est compris entre 49 et 499 personnes avant la croissance externe. Il s'agit d'un effectif consolidé lorsque les entreprises sont structurées avec une 
holding de contrôle. Concernant la position de preneur d'ordres, il a été demandé aux MEP sondées de préciser elles-mêmes si elles sont sous-traitantes ou non (il s'agit d'une des questions du questionnaire posté) ${ }^{1}$.

Dans une première partie, une recension de la littérature permet d'établir les principales variables qui entreront dans la construction du questionnaire. Par souci de concision, l'étude exploratoire préliminaire et les différents tests qualitatifs du questionnaire ne sont pas exposés. La deuxième partie est consacrée à la présentation de l'étude empirique et des résultats. La dimension hétéronome de l'action stratégique des MEP sous-traitantes est discutée dans une troisième partie. En conclusion sont présentés les apports, les limites de ce travail ainsi que les axes de recherche futures.

\section{Approche théorique des choix de croissance externe en MEP}

\subsection{Le dirigeant-propriétaire au cœur de la prise de décision stratégique}

Dans une MEP, les motivations de la croissance externe, la perception des obstacles pouvant déboucher sur l'abandon d'un projet, les difficultés éprouvées, le type d'opération, leur nombre, le déroulement du processus d'acquisition dépendront en grande partie de la personnalité lato sensu du dirigeant-propriétaire. Par personnalité, nous entendons son vécu, son système de valeur ou ses aspirations, son attitude en tant que chef d'entreprise - ses pratiques de gestion -, en d'autres termes, tous les éléments permettant de déterminer un «profil», en référence aux travaux sur les typologies d'entrepreneurs. Parmi ces travaux, notons que peu associent explicitement le mode de croissance envisagé avec les caractéristiques des dirigeants-propriétaires. L'association se fait plus avec l'attitude à l'égard de la croissance : tendance à l'envisager favorablement ou à la rejeter. Après avoir fait un recensement de cette littérature depuis 1967, date des travaux originaux de Smith, nous avons retenu un certain nombre de variables ayant déjà été définies et utilisées dans des travaux de référence. Nous nous proposons de les regrouper en trois catégories : variables de vécu, de pratiques de gestion, d'aspirations relativement à la croissance (tableau 1).

1. En référence à la définition adoptée par le Conseil économique et social français dans un rapport publié au Journal officiel du 26 avril 1973. On peut également se référer à la définition, plus précise mais plus restrictive, de la Loi no 75-1334 du 31 décembre 1975. 


\section{TABLEAU 1}

Variables de profil des dirigeants-propriétaires

\begin{tabular}{|c|c|c|}
\hline $\begin{array}{l}\text { Groupes de } \\
\text { variables }\end{array}$ & $\begin{array}{l}\text { Variables permettant d'établir } \\
\text { le profil des dirigeants-propriétaires }\end{array}$ & $\begin{array}{l}\text { Recherches de } \\
\text { référence }\end{array}$ \\
\hline \multirow{3}{*}{$\begin{array}{l}\text { Nominales binaires } \\
\text { ou multimodales }\end{array}$} & $\begin{array}{l}\text { - Type et niveau de formation initiale: } \\
\text { - autodidacte; } \\
\text { - formation supérieure, en gestion, ingénieur, } \\
\text { autre. }\end{array}$ & $\begin{array}{l}\text { Smith (1967) } \\
\text { Dunkelberg et } \\
\text { Cooper (1982) }\end{array}$ \\
\hline & $\begin{array}{l}\text { - Existence d'une expérience professionnelle } \\
\text { antérieure et nature de cette expérience: } \\
\quad \text { - fonction commerciale; } \\
\text { - fonction production et technique; } \\
\text { - gestion comptable et financière; } \\
\text { - gestion des ressources humaines; } \\
\text { - fonction de direction, autre. }\end{array}$ & $\begin{array}{l}\text { Julien et Marchesnay } \\
\text { (1987) }\end{array}$ \\
\hline & $\begin{array}{l}\text { - Type d'entreprise dans laquelle elle a eu lieu: } \\
\text { - grande entreprise; } \\
\text { - PME / PMI; } \\
\text { - dans ce qui allait devenir leur propre entreprise. } \\
\text { - } \\
\text { Mode d'accession à la direction: } \\
\text { - par création; } \\
\text { - par rachat; } \\
\text { - par héritage. }\end{array}$ & $\begin{array}{l}\text { Lafuente et Salas } \\
\text { (1989) }\end{array}$ \\
\hline $\begin{array}{l}\text { Variables } \\
\text { de pratiques } \\
\text { de gestion }\end{array}$ & $\begin{array}{l}\text { - Capital ouvert / «fermé» à des actionnaires } \\
\text { extérieurs. } \\
\text { - Existence / absence d'outils de planification. }\end{array}$ & $\begin{array}{l}\text { Smith (1967) } \\
\text { Lorrain et Dussault } \\
\text { (1988) }\end{array}$ \\
\hline $\begin{array}{l}\text { Nominales } \\
\text { binaires }\end{array}$ & $\begin{array}{l}\Rightarrow \text { Organisation structurée ou non } \\
\text { avec une holding. }\end{array}$ & $\begin{array}{l}\text { Julien et Marchesnay } \\
\text { (1987) }\end{array}$ \\
\hline $\begin{array}{l}\text { Variables } \\
\text { d'aspirations } \\
\text { relative à la } \\
\text { croissance }\end{array}$ & $\begin{array}{l}\text { - Aspirations « personnelles », dans une logique } \\
\text { plutôt « opportuniste »* ou CAP (par ordre de } \\
\text { priorité : croissance, autonomie, pérennité)** } \\
\text { - développer l'entreprise, la faire croître; } \\
\text { - valoriser ses compétences d'entrepreneur; } \\
\text { - augmenter ses revenus. }\end{array}$ & Davidsson (1989) \\
\hline $\begin{array}{l}\text { Ordinales } \\
\text { à } 7 \text { points }\end{array}$ & $\begin{array}{l}\text { - Aspirations « patrimoniales », dans une logique } \\
\text { plutôt «artisan »* ou PIC (par ordre de priorité : } \\
\text { pérennité, indépendance, croissance)** : } \\
\text { - assurer l'avenir de sa famille ; } \\
\text { - pouvoir transmettre un patrimoine important } \\
\text { - la croissance n'est pas une priorité (une finalité), } \\
\text { c'est une nécessité. }\end{array}$ & $\begin{array}{l}\text { Dunkelberg et } \\
\text { Cooper (1982) } \\
\text { Julien et Marchesnay } \\
\text { (1987) } \\
\text { Kolvereid (1992) }\end{array}$ \\
\hline
\end{tabular}

* En référence aux travaux de Smith (1967).

** En référence aux travaux de Julien et Marchesnay (1987).

Revue internationale P.M.E., vol. 17, $\mathrm{n}^{\circ}$ 1, 2004 
Évidemment, certains problèmes de compétences et de ressources de l'organisation varient en fonction de la personnalité et des objectifs du dirigeantpropriétaire. Ainsi, certains dirigeants, dans une logique patrimoniale dominante, auront pour souci principal de pérenniser l'affaire, de préserver l'indépendance du capital personnel ou familial. Ils répugneront à l'endettement à long terme et à l'accueil de nouveaux actionnaires non familiaux. Le financement de la croissance pourrait alors être problématique. Lorsque le besoin d'indépendance prédomine, la tendance est de s'entourer de peu de collaborateurs et de ne déléguer que les tâches périphériques. Ici, le manque de ressources humaines pourrait également constituer un obstacle. D'autres, au contraire, plus attirés par l'expansion, plus tournés vers l'extérieur et les défis de l'environnement, n'hésiteront pas, par exemple, à ouvrir leur capital à une société de capital investissement pour financer leur stratégie de croissance externe. Ils seront plus enclins à recourir à du conseil extérieur. On peut supposer qu'en cas de besoin ils n'hésiteront pas à solliciter du conseil extérieur pour les aider dans les phases délicates de recherche et d'évaluation de la cible. Toutefois, restera posé le problème de la disponibilité des ressources humaines en interne, afin de permettre l'intégration de la nouvelle entité.

Le niveau d'aversion pour le risque et les aspirations intrinsèques du chef d'entreprise (dirigeant-propriétaire guidé par l'indépendance, par le bien-être de sa famille, par le goût du défi, etc.) auront également une influence importante sur la décision de croissance externe. Dans certains cas, la prudence et l'attentisme domineront, a fortiori si la situation de la MEP est délicate : manque de capitaux propres, de personnel, ratio d'endettement élevé, difficultés à cerner l'avenir, etc.

Enfin, la croissance externe, en agrandissant l'organisation, la complexifie inévitablement. À terme, cette complexité organisationnelle remettra en cause la centralisation au bénéfice d'une plus grande délégation, qui pourrait, dans certains cas, être difficile à accepter et se révéler peu compatible avec l'idéologie dominante. Certains dirigeants-propriétaires préféreront renoncer à un projet de croissance externe et à gérer une organisation plus grande, plus complexe avec toutes les implications que cela aurait sur leur pouvoir. Cependant, ce dernier point est à nuancer si l'organisation est structurée avec une holding de contrôle dans l'optique d'un hypogroupe (Debray, 1997). Il s'agit d'un mode prudent de croissance par constitution de filiales, rachat d'affaires, prises de participation qui constituerait une solution au maintien de la centralisation du pouvoir et au souci de se développer en deçà d'une taille critique.

Ces variables dissuasives, reliées à la personnalité du dirigeant-propriétaire et au potentiel de ressources même de la MEP, sont regroupées dans un tableau intitulé «Difficultés et obstacles pouvant déboucher sur l'abandon d'un projet» (tableau 3). L'étude empirique consistera à déterminer leur force dissuasive pour une MEP sous-traitante ayant à prendre une décision de croissance externe. 


\subsection{Les raisons d'être de la croissance externe à la lumière de la théorie}

Afin de comprendre les raisons pour lesquelles une MEP entreprend une stratégie de croissance externe, il convient, tout d'abord, de se référer aux écrits théoriques existants. Il s'agit, essentiellement, de travaux consacrés aux grandes entreprises cotées. Deux courants de recherche ne sont pas pris en compte du fait des spécificités des entreprises étudiées. Il s'agit des explications fondées sur la théorie managériale et les relations d'agence entre actionnaires et dirigeants. En effet, les MEP se caractérisent par une confusion de la direction et de la propriété ${ }^{2}$. Les explications considérant la valeur de marché des entreprises et les problèmes d'asymétrie d'information sur les marchés financiers ne sont pas considérées non plus, car les cibles, tout comme les MEP initiatrices, ne sont pas cotées.

La première théorie à retenir notre attention est la théorie de l'efficience, selon la dénomination de Trautwein (1990); elle considère que les opérations de croissance externe sont entreprises pour obtenir des effets synergiques. Dans la littérature consacrée à la croissance externe, nous retrouvons toujours les trois types de synergies suivants (Husson, 1987; Trautwein, 1990): les synergies financières, managériales et opérationnelles. Concernant les synergies opérationnelles, elles résultent des rendements ou économies d'échelle, que ce soit en production, R-D, commercialisation, achats ou logistique. Ces économies d'échelle sont la motivation la plus couramment avancée par les dirigeants ou les spécialistes en fusions / acquisitions (Walter et Barney, 1990) pour expliquer la croissance externe, en particulier, les opérations horizontales. Toutefois, la justification de la croissance externe par la recherche de synergies est débattue : selon Porter (1992) «l'échec de la synergie vient plus de l'incapacité des firmes à la comprendre et à la mettre en œuvre, que de quelque vice irrémédiable». Il s'agira dans l'étude empirique qui suit de répondre à trois interrogations majeures :

- dans quelle mesure et dans quels cas la croissance externe des MEP, en particulier des MEP sous-traitantes, est-elle motivée par la recherche d'un effet synergique?

- quel type de synergie est le plus recherché ?

- l'évaluation du potentiel de synergies futures avec l'entreprise cible constitue-t-elle un obstacle important à la réalisation de leur stratégie de croissance externe?

2. En ce qui concerne les entreprises familiales, soulignons que nous avons pris le soin d'éliminer de notre échantillon celles dont la direction est assurée par un dirigeant extérieur à la famille, même s'il est nommé par la coalition au pouvoir. 
Une autre justification de la croissance externe, souvent citée en complément de la théorie de l'efficience, consiste à souligner l'effet des fusions / acquisitions en termes d'accroissement du pouvoir de marché. Ce pouvoir de marché est recherché pour trois raisons principales :

- accroître son pouvoir de négociation au regard de son environnement (Porter, 1992), de ses fournisseurs, clients, créanciers, tiers, etc.;

- mieux gérer sa relation de dépendance avec son environnement (Pfeffer et Salancik, 1978);

- réduire la concurrence.

De nombreuses recherches réalisées en France sur de grandes entreprises ont montré que la croissance externe constitue une réponse à l'internationalisation et un moyen d'accéder rapidement à une taille jugée optimale, du moins suffisante, pour faire face à la compétition européenne, voire mondiale (Allouche, 1981 ; Pène, 1988; Galiegue, 1992). Dans certains cas, résoudre un problème de taille par simple croissance interne serait difficile, car il suppose « un saut» rapide que seule la fusion ou le rachat pourrait permettre. Dès 1974, Gélinier et Gaultier (1974) considéraient que l'acquisition de filiales à l'étranger, afin de devenir « un petit multinational», était d'une utilité majeure pour les moyennes entreprises. Les nouveaux enjeux européens ont intensifié l'intérêt pour la stratégie de croissance externe des moyennes entreprises : «dans les 10 ans qui viennent, les PME européennes vont se restructurer et cela a déjà largement commencé. Le problème de la taille critique sur leurs segments de marché devenus européens est un problème vital. Elles auront un immense besoin de capitaux propres pour y faire face, notamment pour procéder aux acquisitions d'entreprises concurrentes ou complémentaires qui permettront de changer de taille» (Borderie, 1998).

Les secteurs parvenus à maturité ou ceux caractérisés par l'atomisation et une forte intensité concurrentielle laisseraient aussi présager des regroupements futurs entre entreprises de petite et moyenne dimensions. Ainsi, une note d'information de la Direction des transports terrestres français explique: «le transport routier de marchandises est caractérisé par l'existence de milliers de petites, voire de très petites entreprises. Ces entreprises n'ont pas la maîtrise du fret, se trouvent de plus en plus reléguées dans la fourniture, le plus souvent en sous-traitance, de prestations à faible valeur ajoutée, telle la traction. Le rapprochement ou le regroupement volontaire avec d'autres entreprises peuvent être un moyen de contrecarrer cette évolution défavorable ${ }^{3} »$. D'une façon générale, il conviendra de vérifier, avec l'étude empirique, dans quel cas et dans quelle mesure la croissance externe des MEP sous-traitantes est motivée par la recherche d'un pouvoir de marché plus grand.

3. Note d'information de la Direction des transports terrestres n ${ }^{\circ}$ 129, mars-avril 1993. 
L'objectif de diversification constitue une autre justification de la croissance externe ${ }^{4}$. En tenant compte de la position concurrentielle actuelle de l'entreprise, de l'attrait du secteur d'activité actuel et du degré de nécessité du changement, Détrie, Mercier et Ramanantsoa (1982) définissent quatre logiques stratégiques de diversification, que nous nous proposons de reprendre pour analyser le cas particulier d'une MEP. A priori, pour ce type d'entreprise, il y a lieu de penser que la logique de diversification de «placement» sera très peu présente : la croissance externe conglomérale apparaît beaucoup plus comme une stratégie de grands groupes avec des liquidités excédentaires. En revanche, les logiques de «redéploiement» ou de «survie», où le degré de nécessité du changement est plus marqué, pourraient constituer une part non négligeable des opérations de diversification non reliée, entreprises par les MEP de notre échantillon, en particulier, les MEP sous-traitantes. Un élément spécifique fonde notre supposition : en France, d'après les données du ministère de l'Économie, des Finances et de l'Industrie ${ }^{5}$ à la fin des années 1990, pour près d'un preneur d'ordres sur deux, les trois premiers clients représentent plus de $50 \%$ du chiffre d'affaires. Pour $24 \%$ des preneurs d'ordres, le poids des trois premiers clients est égal ou supérieur à $75 \%$ de leur chiffre d'affaires. Ainsi, l'objectif d'une diversification dans le but d'échapper à une dépendance client trop importante pourrait constituer une motivation importante de certaines MEP, en particulier celles travaillant dans la sous-traitance de capacité. Si, pour se diversifier, elles choisissent la croissance externe, a priori plus risquée que la croissance interne ${ }^{6}$, c'est probablement parce qu'il y a « une rapidité de mouvement impérative » (Ansoff, 1989, p. 174). La dernière logique relevée par les auteurs est celle de «confortement» de la position des activités existantes. On trouve dans cette logique de nombreuses intégrations verticales. L'étude empirique déterminera si le souci d'assurer son approvisionnement ou sa distribution sont des motivations majeures pour des dirigeants-propriétaires de MEP sous-traitantes.

La théorie du processus est la dernière analyse issue des travaux sur les grandes entreprises que nous retenons (Jemison et Sitkin, 1986; Guieu, 1994). Selon cette théorie, l'opération de croissance externe n'est pas un choix rationnel (au sens de la rationalité substantielle): elle est la résultante d'un processus décisionnel gouverné par l'ambiguïté, par les jeux politiques entre membres de l'organisation et intervenants extérieurs influençant le processus par des tactiques et des ajustements mutuels.

4. Pour une revue de littérature détaillée sur ce thème, on peut se référer à la thèse de doctorat de Boissin (1994).

5. Source : 4 pages SESSI, $n^{\circ} 65$ et $n^{\circ} 72$.

6. En effet, $71 \%$ des diversifications par acquisition sont des échecs contre $66 \%$ des diversifications par croissance interne. 
Notre préoccupation, dans l'étude empirique, sera de déterminer si, lors du processus de préacquisition, des divergences de vues avec l'une des parties prenantes de son entreprise ou de la cible constituent des facteurs dissuasifs forts pour le dirigeant-propriétaire. Afin d'affiner notre réflexion, nous verrons également s'il avait d'autres choix de développement à l'étude avant de décider de s'engager dans le processus de préacquisition.

Au total, l'analyse des travaux sur les théories explicatives de la croissance externe, ainsi que de ceux liés aux profils d'entrepreneurs nous permet de définir 30 variables incitatives pour une stratégie de croissance externe (tableau 2) et 18 variables dissuasives (tableau 3). Elles sont toutes mesurées par une échelle ordinale de 7 points, de «négligeable » à « primordiale» avec un point « sans objet ».

\section{TABleau 2}

\section{Motivations pour une stratégie de croissance externe}

\footnotetext{
1. Pour élargir votre gamme de produits ou services.

2. Pour élargir votre marché géographiquement.

3. Pour diversifier votre clientèle, afin d'échapper à une forte dépendance.

4. Pour diversifier votre clientèle, à la suite d'une baisse d'activité structurelle sur certains marchés.

5. Pour pénétrer dans un nouveau secteur / marché.

6. Pour bénéficier d'un effet de synergie au plan commercial.

7. Pour bénéficier d'un effet de synergie sur le plan financier.

8. Pour bénéficier d'un effet de synergie en production.

9. Pour bénéficier d'un effet de synergie en potentiel de recherche et d'innovation.

10. Pour atteindre une taille critique au plan régional.

11. Pour atteindre une taille critique au plan national.

12. Pour atteindre une taille critique au plan européen.

13. Pour atteindre une taille critique au plan mondial.

14. Pour acquérir un élément manquant de votre stratégie : une technologie.

15. Pour acquérir un élément manquant de votre stratégie : un savoir-faire.

16. Pour acquérir un élément manquant de votre stratégie : un réseau de distribution.

17. Pour acquérir un élément manquant de votre stratégie : une logistique.

18. Pour éviter le contrôle de la cible par un concurrent.

19. Pour vous maintenir au rythme de croissance du marché.

20. Pour assurer votre approvisionnement.

21. Pour assurer votre distribution.

22. Pour accroître votre pouvoir de négociation avec vos fournisseurs.

23. Pour accroître votre pouvoir de négociation avec vos clients.

24. Pour accroître votre pouvoir de négociation avec vos banquiers.

25. Dans l'espoir d'une revente avec plus-value.

26. Pour assurer, à terme, la survie même de votre entreprise.

27. Car vous envisagiez, à terme, d'être coté en Bourse.

28. Parce qu'une opération de croissance externe est plus rapide à réaliser que la croissance interne.

29. Parce qu'une opération de croissance externe est plus facile à réaliser que la croissance interne.

30. Parce qu'une opération de croissance externe coûte moins cher.
} 


\section{TABLEAU 3 \\ Difficultés et obstacles reliés à l'adoption d'une stratégie de croissance externe}

1. Difficultés pour découvrir la cible.

2. Difficultés pour estimer la valeur de la cible.

3. Difficultés pour estimer les coûts de restructurations ultérieures.

4. Difficultés pour évaluer le potentiel de synergies futures avec la cible.

5. Mésentente préalable à la signature, avec le dirigeant et / ou la famille de la cible.

6. Divergences de vues sur cette opération avec votre famille.

7. Divergences de vues sur cette opération avec vos associés.

8. Divergences de vues sur cette opération avec votre capital investisseur.

9. Divergences de vues sur cette opération avec votre banquier.

10. Difficultés pour financer l'opération.

11. Difficultés pour financer les restructurations après l'opération.

12. Difficultés relationnelles avec la nouvelle équipe.

13. Manque de personnel pour manager la nouvelle équipe.

D’une façon plus générale, variables dissuasives pour le dirigeant-propriétaire:

14. Avenir très imprévisible, donc prudence et attentisme.

15. Difficultés pour cerner le délai de retour sur investissement de l'opération.

16. Situation financière de votre entreprise fragile (trop endettée, manque de capitaux propres, etc.), donc prudence.

17. Crainte d'une perte de contrôle à cause d'une dilution de votre capital pour financer l'opération.

18. Appréhension suscitée par le changement de dimension, le management d'une organisation plus grande.

Après ces approches générales, il convient de resituer la MEP sous-traitante dans son environnement. Nous nous focalisons donc sur les nouvelles politiques achats des grands donneurs d'ordres pour cerner leur influence sur les choix stratégiques des sous-traitants.

\subsection{Influence des nouvelles politiques des donneurs d'ordres sur la stratégie des MEP sous-traitantes}

Un profond bouleversement dans le domaine manufacturier, en particulier, dans le secteur automobile, a eu lieu au début des années 1990. Nous considérons ce secteur, car, historiquement à l'avant-garde, il a innové dans de nombreuses méthodes de gestion de production, de management des achats et approvisionnements, ayant ensuite servi de références dans de nombreux autres domaines industriels (Bigras et Bourque, 1999).

À cette période, les plus grands constructeurs occidentaux sont passés d'un mode de «production de masse » à une logique de «lean production», production au plus juste (Womack, Jones et Ross, 1992). Ce type de production vise à améliorer continuellement la qualité (principe de qualité totale), tout en réduisant le coût par unité et en fournissant une plus grande variété de produits en un minimum 
de temps (principe du juste à temps). Dans cette logique, l'ingénierie simultanée est développée. Sur un projet particulier, il s'agit d'intégrer, du moins de coordonner étroitement la R-D, la conception, la planification, la fabrication et le marketing dans le principal but de réduire le temps de développement des nouveaux produits et d'innover, grâce à l'échange continu d'informations, entre les experts des différents domaines. Développée dans un souci d'efficience interne, l'ingénierie simultanée s'étend peu à peu aux sous-traitants, qui sont intégrés aux projets dès la phase de conception (Lamming, 1993).

Pour réduire le temps d'assemblage sur les chaînes de montage et dans un souci de flexibilité de la production, l'assemblage modulaire est effectué sur des plates-formes communes. La modularisation contribue à des conceptions fondées sur des logiques d'assemblages de sous-ensembles du produit final par des soustraitants de spécialité. Le fournisseur devient responsable depuis la phase de design, en passant par le développement et la production jusqu'à l'assemblage, de la qualité et de la fiabilité d'un système complet (Ali, Smith, Saker, 1997). Dans cette logique, les donneurs d'ordres, au lieu d'avoir un nombre élevé de fournisseurs sélectionnés sur le seul critère de prix, recherchent désormais quelques interlocuteurs privilégiés. On aboutit à une configuration « en gradins » entre donneurs d'ordres et sous-traitants, où seuls les fournisseurs de premier rang sont en contact direct avec le donneur d'ordres (Hines, 1995).

Enfin, dans un environnement en juste à temps, la réduction du nombre de fournisseurs est plus marquée (St John et Heriot, 1993). On tend vers le «single » ou le «dual sourcing» (Zeng, 2000), ce qui engendre à terme une diminution de la base fournisseurs. Ford, par exemple, dans le cadre du projet Ford 2000, avait annoncé une baisse de $90 \%$ de ses fournisseurs de premier rang.

Quelles sont les conséquences pour les sous-traitants de moyenne dimension?

De la diminution de la base fournisseurs résulte, comme le constate Hyun (1994), une tendance vers la concentration industrielle, que ce soit pour les fournisseurs de premier rang, les équipementiers ou les sous-traitants des rangs inférieurs. Ainsi, un sous-traitant de moyenne dimension se doit d'atteindre une certaine taille pour pouvoir être référencé ou maintenir sa position de «fournisseur privilégié » ou «preferred supplier». Il s'agit d'un fournisseur partenaire, le plus souvent intégré dès la phase de conception dans une logique de co-développement, capable de s'engager dans une démarche de progrès continu pour assurer une qualité totale, une diminution régulière de ses coûts et de ses délais - délais de développement mais également de livraison (Turnbull, Oliver et Wilkinson, 1992 ; Dorsch, Swanson et Kelly, 1998).

Les pratiques de juste à temps contribuent aussi à expliquer cette nécessité pour les sous-traitants d'avoir une taille critique pour au moins les trois raisons suivantes :

Revue internationale P.M.E., vol. 17, nº 1, 2004 
1. Le fournisseur doit être en mesure de réaliser les investissements nécessaires en termes de compatibilité des systèmes d'information et de synchronisation des processus de production avec son donneur d'ordres afin d'assurer un « zéro délai » (Spekman, Kamauff et Spear, 1999) : mise en place d'un EDI (échange de données informatisé) interfaçage des systèmes d'information avec un serveur Web, nécessité d'avoir au minimum un système MRP (Materials Requirements Planning), voire un progiciel de gestion intégrée, etc. Des investissements sont aussi souvent nécessaires pour refondre son organisation, afin de mettre en place un système Kanban de gestion des flux internes et introduire du Kaizen dans ses ateliers afin de promouvoir l'amélioration continue de la qualité. Enfin, dans cette logique de qualité totale, les constructeurs exigent de leurs fournisseurs qu'ils s'alignent sur leurs propres normes. Or, une certification représente un coût important pour une moyenne entreprise.

2. Le fournisseur doit pouvoir garantir des volumes de production plus importants et être à même d'assurer des livraisons fréquentes et ponctuelles.

3. Enfin, pour respecter les délais d'approvisionnement et limiter les coûts de transport (résultant notamment de la multiplication des commandes ouvertes), les donneurs d'ordres vont choisir leurs fournisseurs selon leur capacité de s'implanter au plus près de leurs chaînes de montage et de les accompagner, le plus rapidement possible, dans leur développement à l'international. Le développement des « districts industriels » est au cœur de cette logique.

Enfin, avec la modularisation de la production, l'impératif pour les soustraitants consiste à maîtriser d'autres technologies, souvent proches ou complémentaires, pour leur permettre de proposer des prestations plus complètes ou pour être à même de réaliser des sous-ensembles. L'objectif est d'apporter plus de valeur ajoutée au donneur d'ordres en devenant un «intégrateur». À partir d'une analyse de ces stratégies modulaires dans les secteurs informatique et automobile, Sako et Murray (2000) observent que devant les pressions émanant des constructeurs, les sous-traitants se regroupent afin de créer une base plus large et plus solide de connaissances techniques et de ressources financières. Une étude auprès de 25 PME sous-traitantes de l'industrie automobile en Italie montre également que $60 \%$ de ces entreprises ont opté pour des rachats d'autres PME afin de satisfaire aux exigences de valeur ajoutée de leurs donneurs d'ordres (Calabrese, 2000).

De cette analyse des relations entre donneurs d'ordres et sous-traitants, le tableau 2 est complété par six nouvelles variables de motivations pour une stratégie de croissance externe (tableau 4). 


\section{TABLEAU 4}

\section{Motivations pour une stratégie de croissance externe}

31. Pour accompagner vos donneurs d'ordres dans leur développement national ou international

32. Pour être à même de fournir vos donneurs d'ordres en juste à temps.

33. Pour être à même d'offrir des capacités de production plus importantes à vos donneurs d'ordres.

34. Pour être à même d'offrir des prestations plus complètes.

35. Pour devenir fournisseur de premier rang ou référencé.

36. Pour conserver cette position.

En conclusion, l'analyse des aspirations des dirigeants-propriétaires, du potentiel de ressources internes de l'entreprise et des exigences de l'environnement permet de relever un certain nombre de variables incitatives et dissuasives des stratégies de croissance externe des MEP. L'objet de l'étude empirique est de tenter de comprendre ces stratégies en hiérarchisant ces variables et en déterminant l'influence du profil des dirigeants-propriétaires.

\section{Présentation de l'étude empirique et des résultats}

\subsection{Constitution et description de l'échantillon}

L'enquête a été réalisée par l'envoi d'un questionnaire auprès de 994 dirigeantspropriétaires de MEP françaises, tous secteurs confondus, ayant réalisé au moins une opération entre 1992 et 1999. Ce questionnaire comprend cinq parties portant sur les motivations de la croissance externe, les difficultés rencontrées et les obstacles pouvant susciter l'abandon d'un projet, les caractéristiques de la MEP et de la cible (mesurées par des variables quantitatives et nominales), le dirigeantpropriétaire : son vécu, ses pratiques de gestion et ses aspirations.

La fiabilité des échelles de mesures utilisées a été testée par le $\rho$ de Jöreskog : pour toutes nos échelles, les $\rho$ sont supérieurs à 0,9 .

Au total, 147 questionnaires sont exploitables. Notons que les données descriptives de l'échantillon ne sont pas représentatives de l'ensemble des MEP françaises. Le chiffre d'affaires moyen de ces $147 \mathrm{MEP}$, sur la période considérée, est de $243 \mathrm{MF}$ (médiane =125 MF), l'effectif moyen de 285 personnes (médiane $=150$ ) et $49 \%$ d'entre elles sont dans une position de sous-traitantes, soit 72 entreprises.

Sur ces 72 entreprises sous-traitantes, 61 sont dans l'industrie et 11 dans le tertiaire. Dans ce dernier cas, il s'agit exclusivement de services aux entreprises : transport-logistique en majorité et quelques sociétés de services en informatique et ingénierie. Les secteurs d'activité des donneurs d'ordres des MEP du tertiaire sont très variés; aucune dominante ne se dégage. Dans l'industrie, les MEP sont 
en majorité dans le travail des métaux (33,3\% du total des entreprises soustraitantes). Sous cette dénomination sont regroupées des entreprises dans le décolletage, la forge, l'emboutissage, l'estampage, le découpage, la chaudronnerie et le traitement des métaux. La transformation des matières plastiques représente $20 \%$ des sous-traitantes, la fabrication d'équipements électriques $8,3 \%$, la fabrication de machines-outils et moules $6 \%$. Les donneurs d'ordres des MEP dans l'industrie sont en majorité des équipementiers ou constructeurs automobiles. Nous trouvons également quelques grands constructeurs de matériels électriques.

Pour ce qui est de l'actionnariat, 45 des entreprises sous-traitantes sont personnelles et 27 familiales.

\subsection{Hiérarchisation des variables de motivation pour la croissance externe}

Pour déterminer dans quelle mesure le fait d'être une MEP sous-traitante ou non (variable dépendante dichotomique) influence la nature et l'importance des motivations pour la croissance externe (36 variables explicatives relevées en première partie), une régression logistique binaire est réalisée avec une procédure séquentielle «pas à pas » ascendante, rapport de vraisemblance (tableau 5). Avec la méthode pas à pas, seules les variables explicatives améliorant la fonction du maximum de vraisemblance sont retenues.

\section{TABLEAU 5}

\section{Motivations des MEP sous-traitantes}

Pourcentage global d'individus bien classés

$\mathrm{R}^{2}$

Variables dans l'équation finale et coefficients associés
$92,52 \%$

0,972

La croissance externe est plus rapide que la croissance interne: 13,3325 .

Offrir des capacités de production plus importantes à ses donneurs d'ordres : 10,0987.

Offrir des prestations plus complètes : 4,6697.

Pour devenir fournisseur de premier rang ou privilégié : 1,8715 .

Constante : $-1,9196$.

Nombre d'observations sélectionnées : 147. 
Le $\mathrm{R}^{2}$ de McFadden mesure l'amélioration du logarithme de la fonction de vraisemblance permise par l'introduction des variables explicatives autres que la constante ${ }^{7}$.

La rapidité de la croissance externe, comparativement à un développement interne, est la motivation ayant le coefficient positif le plus élevé. Il s'agit donc de l'explication la plus caractéristique d'une MEP en position de sous-traitance comparativement à une MEP non sous-traitante. Quelle que soit la finalité, la vélocité de la réponse aux exigences des clients est impérative : c'est une condition suspensive de la relation entre donneurs d'ordres et fournisseurs. On comprend que cette vitesse de réaction soit capitale, lorsqu'il y a menace d'un «déréférencement» ou lorsque le donneur d'ordres est dans une logique de réduction de sa base de fournisseurs.

La deuxième priorité des MEP sous-traitantes, accordée à l'augmentation des capacités de production, correspond bien à une situation où les donneurs d'ordres entreprennent une rationalisation de leur base de fournisseurs.

La motivation suivante «offrir des prestations plus complètes » est symptomatique de la tendance à l'achat de sous-ensembles, de modules mettant en œuvre plusieurs technologies ou savoir-faire. Pour vivre, les petites et moyennes structures sont alors obligées de toucher à d'autres spécialités, que ce soit dans la sous-traitance industrielle ou dans le service. Pour une MEP dans le transport, par exemple, ne proposer que de la traction est une stratégie risquée. Désormais, les clients attendent de leurs fournisseurs de savoir gérer aussi bien les flux physiques que les flux d'informations. Il s'agit de maîtriser des compétences en gestion de plates-formes logistiques. Dans le service en informatique et ingénierie, la tendance est la même : les grands comptes souhaitent avoir pour interlocuteurs des entreprises capables de leur proposer des prestations en infogérance, animation de réseaux, développements spécifiques, conseils, formation, etc. Le métier d'une société de services en informatique et ingénierie se définit de plus en plus par l'intégration.

Si la motivation de «devenir fournisseur de premier rang ou privilégié » apparaît en dernier dans l'équation finale, c'est parce que les objectifs cités précédemment («offrir des capacités de production plus importantes», « des prestations plus complètes ») constituent les moyens prioritaires à mettre en œuvre «rapidement », pour espérer parvenir à cette fin. L'autre raison réside dans la nature même de notre échantillon: seules $28 \%$ des 72 entreprises sous-traitantes se déclarent fournisseurs «privilégiés », de premier rang ou déjà référencés.

7. $\mathrm{R}^{2}=1-[\ln (\mathrm{i}) / \ln (\mathrm{o})]$ où $\ln (\mathrm{i})$ est la valeur du logarithme de la fonction de vraisemblance correspondant à l'introduction de la variable explicative et $\ln (\mathrm{o})$ est la valeur maximale du logarithme de la fonction de vraisemblance lorsque tous les coefficients de régression autres que la constante sont nuls. 
Pour affiner l'étude, il convient de se focaliser sur les seules MEP soustraitantes afin de voir en quoi le fait d'être déjà référencées ou non (variable dépendante dichotomique) va influer sur leurs motivations pour la croissance externe (tableau 6).

\section{TABLEAU 6}

\section{Motivations des entreprises sous-traitantes déjà référencées}

\begin{tabular}{ll}
$\begin{array}{l}\text { Pourcentage global d'individus } \\
\text { bien classés }\end{array}$ & $83,33 \%$ \\
$\mathrm{R}^{2}$ & 0,8168 \\
Variables dans l'équation finale & $\begin{array}{l}\text { Atteindre une taille critique européenne : } 0,3961 . \\
\text { et coefficients associés }\end{array}$ \\
& $\begin{array}{c}\text { Accompagner ses donneurs d'ordres dans leur développement } \\
\text { à l'international : } 0,3332 . \\
\text { Constante : }-3,2065 .\end{array}$ \\
\hline
\end{tabular}

Nombre d'observations sélectionnées : 72.

Dès lors que la MEP est déjà homologuée, référencée auprès de grands donneurs d'ordres, son souci principal est de conforter, voire renforcer cette position. Il ne s'agit plus seulement d'être un interlocuteur privilégié sur le sol national: l'enjeu est d'atteindre une taille critique à l'échelle européenne pour pouvoir suivre ses donneurs d'ordres à l'international (tableau 6). Il s'agit, notamment, de répondre aux exigences de juste à temps pour l'approvisionnement en étant à même de proposer des implantations proches des nouvelles unités d'assemblage ou de production des clients.

Les sous-traitants promus au rang de fournisseurs «privilégiés » remplissent déjà un certain nombre de critères tels que la maîtrise de compétences de conception, mais aussi de validation. Cela sous-entend qu'ils ont dû mettre en place des ressources humaines et techniques spécifiques et qu'ils disposent déjà d'une taille suffisante pour absorber les frais inhérents à ces investissements. Ainsi, Calabrese (2000), dans son étude sur 25 PME sous-traitantes de l'industrie automobile en Italie, montre qu'il existe une forte corrélation positive entre la taille du soustraitant et le degré de maturité de sa relation avec son donneur d'ordres.

Nous faisons le même constat dans notre étude. Les MEP sous-traitantes référencées ont un chiffre d'affaires moyen (avant opération de croissance externe) plus important que les MEP sous-traitantes non référencées : le test de Fisher de l'Anova est significatif à $5 \%(\mathrm{~F}=4,177$ et $\mathrm{S}=0,043)$. Non seulement elles sont de plus grande taille, mais, dans leur souci d'atteindre une taille critique, elles effectuent aussi des opérations de plus grande envergure : le chiffre d'affaires moyen des cibles des sous-traitantes référencées est plus important que celui des sous- 
traitantes non référencées (test de Fisher de l'Anova significatif à 5\%, F=5,322 et $S=0,024$ ). Le constat est le même pour l'effectif moyen de la cible (test de Fisher de l'Anova significatif à $5 \%, F=4,779$ et $\mathrm{S}=0,032$ ).

En synthèse, la principale explication du choix de la croissance externe, pour les MEP sous-traitantes, réside dans le fait qu'elle leur apparaît comme une réponse plus rapide aux contraintes environnementales, comparativement à une solution interne. Ces contraintes correspondent ici aux nouvelles politiques achatsapprovisionnement des donneurs d'ordres. Par conséquent, le développement externe constituerait un moyen privilégié par les MEP sous-traitantes pour ajuster, le plus rapidement possible, leur niveau «d'agressivité stratégique » (Ansoff, 1989) avec le niveau de turbulence, d'exigence de leur environnement.

Si nous nous focalisons sur la stratégie des MEP référencées, comparativement aux non référencées, c'est la dimension internationale qui prime. Nous observons, par ailleurs, que pour conserver leur rang de fournisseur "privilégié », les MEP migrent progressivement d'un statut de moyenne entreprise vers un statut de grande entreprise.

L'analyse des facteurs dissuasifs va nous permettre de mieux comprendre leur position en ce qui concerne la croissance externe.

\subsection{Hiérarchisation des variables dissuasives de la croissance externe}

Tout comme pour l'analyse des motivations, une régression logistique est réalisée : la variable dépendante dichotomique est le fait d'être une MEP sous-traitante ou non; les variables explicatives sont les 18 variables de difficultés et d'obstacles relevées en première partie.

\section{TABLEAU 7}

\section{Obstacles, freins à la croissance externe des MEP sous-traitantes}

\begin{tabular}{ll}
\hline $\begin{array}{l}\text { Pourcentage global d'individus } \\
\text { bien classés }\end{array}$ & $57,82 \%$ \\
$\mathrm{R}^{2}$ & 0,6779 \\
Variables dans l'équation finale & Crainte de perte de contrôle : 0,2302. \\
et coefficients associés & Constante : $-0,5423$. \\
\hline
\end{tabular}

Nombre d'observations sélectionnées : 147.

Une seule variable apparaît dans l'équation finale: pour les MEP soustraitantes, le seul et principal frein à une stratégie de croissance externe est la crainte de perdre le contrôle à cause d'une dilution du capital pour financer cette 
stratégie (tableau 7). Nous comprenons que les valeurs patrimoniales de la coalition au pouvoir sont déterminantes. Même si certaines MEP sous-traitantes de notre échantillon ont ouvert leur capital à une société de capital investissement minoritaire, elles préféreront renoncer à un projet de croissance externe s'il risque de réduire leur contrôle sur l'entreprise. Il y a donc conflit dans ces entreprises entre des aspirations patrimoniales et des impératifs stratégiques de croissance pour satisfaire les donneurs d'ordres.

Cette stratégie constitue un dilemme. En effet, pour être ou se maintenir référencées, elles se doivent d'être dans une logique de croissance, logique qui aboutira, à plus ou moins longue échéance, à une limitation de leur indépendance financière, alors que les aspirations de la coalition au pouvoir sont clairement de conserver le contrôle. Aussi, il semble que cette stratégie de croissance externe s'appréhenderait davantage comme une stratégie hétéronome que comme une stratégie délibérée. Certes, le dilemme entre croissance et maintien du contrôle personnel ou familial trouverait une réponse dans la constitution d'une holding. On observe que $82 \%$ des MEP sous-traitantes sont structurées avec une holding de contrôle contre $65,3 \%$ des MEP non sous-traitantes : le test du chi carré est significatif à $5 \%\left(\chi^{2}=5,200\right.$ et $\left.\mathrm{S}=0,023\right)$.

Notre interrogation, dès lors, porte sur les motivations de la constitution de cette holding. Dans l'idée d'un hypogroupement, évoqué en première partie, est-ce pour « faciliter la gestion du groupe, car les filiales sont des entités distinctes » et pour «pouvoir conserver des unités de petites tailles»? Dans une optique plus patrimoniale, est-ce pour « verrouiller le capital », pour « préparer la transmission du groupe », plus particulièrement, pour « bénéficier d'avantages fiscaux » ? Enfin, est-ce pour «faciliter des opérations de croissance externe par l'effet de levier»? Les dirigeants-propriétaires des 147 MEP de l'échantillon ont dû indiquer l'importance de leurs motivations pour chacune de ces six variables sur une échelle ordinale de 7 points. Une régression logistique permet de déterminer la contribution de chacune de ces variables explicatives (tableau 8). Rappelons que la variable dépendante dichotomique est le fait d'être une MEP sous-traitante ou non.

\section{TABLEAU 8}

\section{Motivation des MEP sous-traitantes pour la constitution d'une holding}

$\begin{array}{ll}\begin{array}{l}\text { Pourcentage global d'individus } \\ \text { bien classés }\end{array} & 61,90 \% \\ \mathrm{R}^{2} & 0,7865 \\ \text { Variables dans l'équation finale } & \text { Faciliter les opérations de croissance externe } \\ \text { et coefficients associés } & \text { par l'effet de levier : } 0,2305 . \\ & \text { Constante : }-0,6951 .\end{array}$

Nombre d'observations sélectionnées: 147. 
La principale explication de la constitution d'une holding pour les MEP soustraitantes réside dans la possibilité de faire jouer l'effet de levier pour financer leur stratégie de croissance externe (tableau 8). La holding devient le moyen de concilier les préférences financières de la coalition au pouvoir avec les pressions de croissance exercées par les donneurs d'ordres. En effet, elle constitue une solution au financement de la croissance externe lorsque la MEP ne peut totalement autofinancer l'opération envisagée, lorsque sa coalition au pouvoir ne souhaite pas ouvrir le capital, enfin, lorsque les membres de cette coalition ne peuvent ou ne veulent pas emprunter en leur nom propre pour injecter ensuite des liquidités dans l'entreprise. C'est la holding qui emprunte en son nom pour racheter les actions ou parts de l'entreprise cible. Le régime de l'intégration fiscale (plutôt que celui des sociétés mères) facilite ensuite le remboursement de l'emprunt contracté. Notons que, sur la période considérée, les MEP sous-traitantes ont réalisé, en moyenne, 2,82 opérations.

À ce stade de l'analyse où des antagonismes ont été mis en lumière entre les facteurs incitatifs et dissuasifs des stratégies de croissance externe des MEP soustraitantes, seule l'approche par le profil du dirigeant-propriétaire permettra de faire progresser notre compréhension du phénomène.

\subsection{Influence du profil du dirigeant-propriétaire}

Peu de variables de vécu ou de pratiques de gestion discriminent de façon significative le groupe des MEP sous-traitantes des MEP non sous-traitantes. Le seul point notable est que les dirigeants-propriétaires des entreprises sous-traitantes sont plus nombreux à avoir acquis leurs expériences professionnelles dans des PME/PMI (à 44,5\%) contrairement aux dirigeants-propriétaires des MEP non sous-traitantes issus, en priorité, du monde de la grande entreprise (à 40\%) : le test du chi carré est significatif à $5 \%\left(\chi^{2}=5,358\right.$ et $\left.S=0,050\right)$. Il est donc légitime de penser que les dirigeants-propriétaires des MEP sous-traitantes connaissent très bien l'univers des PME/PMI et ses contraintes inhérentes.

En ce qui concerne leurs aspirations au regard de la croissance, une régression logistique a été réalisée à partir des six variables d'aspirations relevées en première partie ( $c f$. tableau 1).

L'opinion des dirigeants-propriétaires des MEP sous-traitantes sur les finalités guidant leur politique de croissance externe conforte l'idée de stratégie «contrainte ». En effet, d'après eux, «la croissance n'est pas une priorité, c'est une nécessité » (tableau 9) et il s'agit de la seule variable explicative apparaissant dans l'équation finale. Or, dans la majorité des cas, ils ont considéré plusieurs possibilités de développement avant de prendre leur décision : 62,5\% des dirigeantspropriétaires des MEP sous-traitantes affirment qu'au moment des premiers contacts 


\section{TABLEAU 9 \\ Opinion des dirigeants-propriétaires des MEP sous-traitantes au regard de la croissance}

\begin{tabular}{ll}
\hline $\begin{array}{l}\text { Pourcentage global d'individus } \\
\text { bien classés }\end{array}$ & $56,42 \%$ \\
$\mathrm{R}^{2}$ & 0,6523 \\
$\begin{array}{l}\text { Variables dans l'équation finale } \\
\text { et coefficients associés }\end{array}$ & La croissance n'est pas une priorité, c'est une nécessité : 0,3644. \\
& Constante : $-1,8172$. \\
\hline
\end{tabular}

Nombre d'observations sélectionnées : 147.

avec la cible ils avaient un autre choix de développement à l'étude (croissance interne, partenariat, etc.). La stratégie de croissance externe constituerait donc la seule réponse possible, permettant de combiner des critères d'obtention de taille critique nationale ou internationale, de satisfaction des donneurs d'ordres avec un impératif de rapidité.

\section{Analyse et discussion}

Dans quelle mesure les stratégies de croissance externe des MEP sous-traitantes relèvent-elles de stratégies hétéronomes? Telle est la question initialement posée dans cette recherche. Plusieurs points sont à considérer pour tenter d'y répondre. Tout d'abord, la notion d'hétéronomie renvoie à celle d'adaptation. Dans la logique des approches contingentes, les performances des entreprises dépendent de leur capacité d'adaptation. Pour être efficace, une adaptation doit pouvoir être menée à un rythme et connaître une ampleur qui conviennent aux changements environnementaux. L'entreprise doit être en mesure de se transformer à un rythme compatible avec les évolutions, les sautes de son environnement. Notons que si l'inertie au sein de l'entreprise est forte, on parlera alors plutôt d'adoption que d'adaptation (Koenig, 1996).

Cette notion d'adaptation appelle une réflexion sur deux dimensions déterminantes :

- la prépondérance de la vélocité dans la réponse des MEP aux exigences des donneurs d'ordres : c'est le problème du rythme de la réponse qui est posé ;

- et la problématique du choix: avaient-elles réellement le choix entre plusieurs options de développement?

Dans de nombreux travaux, la justification de la croissance externe par le facteur temps, comparativement au développement interne, a le plus souvent été 
proposée pour une stratégie de diversification non reliée (Salter et Weinhold, 1981 ; Teece, 1982), en particulier, une diversification « de survie» ou « de redéploiement impératif », selon la typologie de Détrie, Mercier et Ramanantsoa (1982). Mais, dans notre étude, il n'existe aucun lien statistiquement significatif entre la variable nominale «diversification non reliée » et la variable ordinale de motivation «parce que la croissance est plus rapide à réaliser que la croissance interne » (le test $\mathrm{U}$ de Mann-Whitney n'est pas significatif). Plus généralement, il n'y a pas de lien entre la variable de « direction de la croissance » (variable nominale multiple : diversification reliée, non reliée, croissance horizontale, verticale) et la motivation «parce que la croissance est plus rapide à réaliser que la croissance interne » (le test de Kruskal-Wallis n'est pas significatif). Les MEP n'entreprennent donc pas de diversification par croissance externe, plutôt que par développement interne, parce qu'il y a « une rapidité de mouvement impérative» (Ansoff, 1989, p. 174).

En ce qui concerne la problématique du choix, il ressortait des études françaises de Perez et Gabrié (1972) puis Gabrié (1976), que la majorité des firmes, optant pour la croissance externe, n'avait, en fait, pas toujours le choix entre croissance externe et croissance interne; en particulier, lorsqu'elles sont confrontées à un impératif d'obtention rapide d'une taille compétitive sur un marché ou bien à la nécessité d'une diversification rapide. C'est la raison pour laquelle ces auteurs parlent de stratégies « contraintes».

Si l'impératif de diversification rapide ne semble pas s'appliquer au cas des MEP de notre échantillon, en revanche, celui d'obtention rapide d'une taille compétitive sur un marché est déterminant. Toutefois, la notion de stratégie de «rattrapage réactif» par rapport aux concurrents ne paraît pas appropriée. Cette stratégie relevée par Allouche (1981) émanait d'entreprises françaises en situation défavorable depuis au moins trois ans avant l'opération ; il s'agissait plus d'opérations « défensives » visant à « colmater les brèches du môle». Dans notre étude, le développement externe constitue un moyen privilégié par les MEP sous-traitantes pour ajuster, le plus rapidement possible, leur niveau «d'agressivité stratégique » (Ansoff, 1989) avec le niveau d'exigence de leur environnement.

En synthèse, la prépondérance du critère de rapidité dans le choix d'un développement externe plutôt qu'interne ou conjoint, combiné au fait que la croissance apparaît, pour la majorité des dirigeants-propriétaires, comme une nécessité, laisserait supposer que la croissance externe constitue pour les MEP sous-traitantes une stratégie qui s'impose en raison de l'apparition de transformations profondes dans leur environnement, en l'occurrence les nouvelles politiques achats-approvisionnement des grands donneurs d'ordres.

Mais ne retenir comme démarche stratégique que l'hétéronomie est très discutable, car la réalité est beaucoup plus complexe : d'une part, le mode d'action stratégique d'une entreprise est toujours à la fois le produit d'une intention (dans 
notre étude, la vision des dirigeants-propriétaires, leurs aspirations sont essentielles) et de phénomènes incontrôlés; d'autre part, l'action stratégique est conditionnée par les interactions entre structures, culture et systèmes d'information (Martinet, 1984) expliquant le fonctionnement de toute organisation.

Aussi, notre position est plus nuancée. Il convient d'appréhender la réponse à la question initialement posée le long d'un continuum dont les deux extrémités seraient stratégies hétéronomes et stratégies volontaristes, avec un point «central » pour les stratégies interactives. Dans cette logique, le curseur des stratégies de croissance externe des MEP sous-traitantes se situerait davantage dans le pôle de l'hétéronomie, sans pour autant être à l'extrémité, sur des stratégies contraintes «pures ».

\section{Conclusion}

Les apports de ce travail sont variés. Tout d'abord, un éclairage est effectué sur une manœuvre stratégique très peu étudiée dans le champ de recherche des moyennes entreprises où prévaut, actuellement, un engouement pour des travaux sur les réseaux, les alliances et les partenariats. Lors de la constitution de notre base de données, nous avons pu observer que la croissance externe n'est pas une manœuvre si exceptionnelle pour une moyenne entreprise non cotée que son examen soit dépourvu d'intérêt. D'un point de vue pratique, la mise en lumière de la dimension hétéronome des stratégies de croissance externe des MEP sous-traitantes devrait donner matière à réflexion non seulement aux dirigeants-propriétaires et à leur famille, mais également aux donneurs d'ordres. S'agissant des dirigeantspropriétaires, ils devraient s'interroger sur la pertinence de la création d'une holding, permettant, a priori, de concilier des préférences patrimoniales avec des impératifs stratégiques (Tréhan, 2002). Concernant les donneurs d'ordres, les résultats de ce travail devraient les inciter à réfléchir sur des mesures d'accompagnement, voire de soutien de leurs fournisseurs, susceptibles de devenir «privilégiés ». Les principales limites de l'étude sont d'ordre méthodologique. Compte tenu de la nonreprésentativité de notre échantillon, le problème de la généralisabilité des résultats est soulevé. Par ailleurs, nous sommes consciente que les approches quantitatives comportent des limites en termes de compréhension de la réalité. Aussi, une approche plus qualitative orientée non seulement sur le discours des dirigeantspropriétaires des MEP, mais aussi sur celui des donneurs d'ordres permettrait de compléter et de mieux interpréter les résultats obtenus. Il s'agit d'un axe de développement de notre recherche. 


\section{Bibliographie}

ALI, F., G. SMITH et J. SAKER (1997), «Developing buyer-supplier relationships in the automobile industry », European Journal of Purchasing and Supply Management, vol. $3, n^{\circ} 1$, p. $33-42$.

Allouche, J. (1981), Motifs et efficacité des opérations de croissance externe, Thèse de doctorat, Université Paris IX, Dauphine.

Ansoff, H.I. (1989), Stratégie du développement de l'entreprise, Paris, Les Éditions d'Organisation, $287 \mathrm{p}$.

BIGRAS, Y. et G. BOURQUE (1999), Les nouvelles tendances dans la gestion de la production et dans les relations de travail : le cas de l'industrie nord-américaine de l'automobile, Trois-Rivières, Université du Québec, Cahiers de recherche, septembre, 192 p.

Boissin, J.-P. (1994), Construction d'un cadre d'analyse des déterminants de la performance dans les opérations de diversification par acquisition, Thèse de doctorat, Grenoble, Université Pierre-Mendès-France, ESA, 421 p.

BORDERIE, A. (1998), Financer les PME autrement. Le capital-investissement : des fonds propres pour les entrepreneurs. Une approche française : SIPAREX, Paris, Maxima, Laurent du Mesnil Éditeur, 222 p.

CAlABRese, G. (2000), « Small-medium supplier-buyer relationships in the car industry : evidence from Italy », European Journal of Purchasing \& Supply Management, $\mathrm{n}^{\mathrm{o}} 6$, p. 59-65.

DAVIDSSON, P. (1989), «Entrepreneuship-and after? A study of growth willingness in small firms », Journal of Business Venturing, vol. 4, p. 211-226.

Debray, C. (1997), Structures et stratégies de l'hypogroupe, Thèse de doctorat, Montpellier I, $421 \mathrm{p}$.

DÉRY, R., J.P. DuPUIS, P. Hugron et L. PÉloquin (1993), «La variété des entreprises familiales : Construction théorique et typologique», Cahier de recherche $\mathrm{n}^{\circ}$ 93-8, HEC Montréal.

DÉTRIE, J.P., V. MERCIER et B. RAMANANTSOA (1982), «Cinq règles pour se diversifier », Harvard L'expansion, hiver, p. 37-55.

DoRSCH, M.J., S.R. SWANSON et S.W. KELLY (1998), «The role of relationships quality in the stratification of vendors as perceived by customers », Journal of the Academy of Marketing Science, vol. 26, no 2, p. 128-142.

DunKelberG, W.C. et A.C. COOPER (1982), «Entrepreneurial typologies : an empirical study », Babson College, Frontiers of Entrepreneurship Research, p. 1-15.

ETtingeR, J.-C. (1982), «Some Belgian evidence on entrepreneurial personality», European Small Business Journal, vol. 1, n 2, p. 48-56.

GABRIÉ, H. (1976), Un essai d'explication de la croissance externe des entreprises, Thèse de doctorat en sciences économiques, Université Paris-Dauphine.

GALIEGUE, E. (1992), «La croissance externe des entreprises françaises. Risque majeur ou opportunité historique? », Revue d'économie financière, vol. 22, p. 93-107.

Revue internationale P.M.E., vol. 17, $\mathrm{n}^{\mathrm{o}}$ 1, 2004 
GÉLINIER, O. et A. GAULTIER (1974), L'avenir des entreprises personnelles et familiales : développement-capital-succession-structure, Paris, Éditions Hommes et Techniques, $254 \mathrm{p}$.

GuIEU, G. (1994), Hybridité stratégique et apprentissage organisationnel dans les prises de contrôle d'entreprises, Thèse de doctorat, Université Pierre-Mendès-France, Grenoble II.

HINES, P. (1995), «Network sourcing: a hybrid approach», International Journal of Purchasing and Materials Management, vol. 31, n 2, p. 17-24.

Husson, B. (1987), La prise de contrôle d'entreprises: motivations, conséquences et freins. Une analyse des contextes français et américain, Paris, Presses universitaires de France, $240 \mathrm{p}$.

HYUN, J.-H. (1994), «Buyer-supplier relations in the European automobile component industry », Long Range Planning, vol. 27, n 2, p. 66-75.

JEMISON, D.B. et S.B. SITKIN (1986), «Corporate acquisitions : a process perspective», Academy of Management Review, vol. 11, n 1 , p. 145-163.

JULIEN, P.-A. et M. MARCHESNAY (1987), La petite entreprise : principes d'économie et de gestion, Paris, Vuibert Gestion, 288 p.

KoenIG, G. (1996), Management stratégique : Paradoxes, interactions et apprentissages, Paris, Nathan, 544 p.

KOLVEREID, L. (1992), «Growth aspirations among Norwegian entrepreneurs », Journal of Business Venturing, vol. 7, p. 209-222.

LAFUENTE, A. et V. SALAS (1989), «Types of entrepreneurs and firms : the case of new Spanish firms », Strategic Management Journal, vol. 10, p. 17-30.

LAMming, R.C. (1993), Beyond Partnership : Strategies for Innovation and Lean Supply, New York, Prentice-Hall, 299 p.

LORRAIN, J. et L. DUSSAULT (1988), «Les entrepreneurs artisans et opportunistes : une comparaison de leurs comportements de gestion», Revue internationale PME, vol. $1, \mathrm{n}^{\circ}$ 2, p. $158-171$.

MARTINET, A.C. (1984), Management stratégique: organisation et politique, Paris, McGraw-Hill, coll. «Stratégie et management», 118 p.

PÈnE, D. (1988), «Arbitrage entre croissance interne et externe en France», Revue d'économie financière, $\mathrm{n}^{\text {os }}$ 5-6, p. 112-122.

PEREZ, R. et H. GABRIÉ (1972), L'insertion des opérations de croissance externe dans les stratégies des entreprises, Institut de sciences économiques appliquées, Tome I.

PFEFFER, J. et G.R. SALANCIK (1978), The External Control of Organizations : A Resource Dependence Perspective, New York, Harper \& Row, 300 p.

PORTER, M.E. (1992), L'avantage concurrentiel: comment devancer ses concurrents et maintenir son avance, Peyrieu, InterÉditions, 647 p.

St John, C.H. et K.C. Heriot (1993), «Small suppliers and JIT», Purchasing, hiver, p. 11-16. 
SAKo, M. et F. Murray (2000), «Automobile, informatique et stratégie modulaire», dans L'art de la stratégie, Paris, Village Mondial, 399 p.

SALTER, M.S. et W.A. WEINHOLD (1981), « Choosing compatible acquisitions », Harvard Business Review, vol. 59, no 1, p. 117-127.

SCHWARTZ, S. (1982), «Factors affecting the probability of being acquired: evidence from the United States », The Economic Journal, vol. 92, p. 391-398.

SMITH, N.R. (1967), The Entrepreneur and His Firm: The Relationship between Type of Man and Type of Company, East Lansing, Mich., Michigan State University, Bureau of Business and Economic Research.

SPEKMAN, R.E., J. KAMAUFF et J. SPEAR (1999), « Towards more effective sourcing and supplier management », European Journal of Purchasing \& Supply Management, vol. 5, p. 103-116.

TEECE, D.J. (1982), « Towards an economic theory of the multiproduct firm », Journal of Economic Behavior and Organization, vol. 3, n 1, p. 39-63.

TRAUTWEIN, F. (1990), « Merger motives and merger prescriptions », Strategic Management Journal, vol. 11, p. 283-295.

TRÉHAN, N. (2002), «Usages de la société holding par des entreprises personnelles et familiales non cotées pratiquant la croissance externe », Revue Finance Contrôle Stratégie, vol. 5, nº 2, p. 143-162.

Turnbull, P., N. Oliver et B. Wilkinson (1992), «Buyer-supplier relations in the UK automotive industry : strategic implications of the Japanese manufacturing model », Strategic Management Journal, vol. 13, p. 159-168.

WALTER, G.A. et J.B. BARNEY (1990), « Research notes and communications management objectives in mergers and acquisitions », Strategic Management Journal, vol. 11, p. 79-86.

WOMACK, J.P., D.T. JONES et D. Ross (1992), Le système qui va changer le monde : une analyse des industries automobiles mondiales, Paris, Dunod, 349 p.

ZENG, A.Z. (2000), «A synthetic study of sourcing strategies », Industrial Management and Data Systems, vol. 100, no 5, p. 1-8.

Revue internationale P.M.E., vol. 17, $\mathrm{n}^{\mathrm{o}}$ 1, 2004 